\title{
Association of persistent and transient worsening renal function with mortality risk, readmissions risk, length of stay, and costs in patients hospitalized with acute heart failure
}

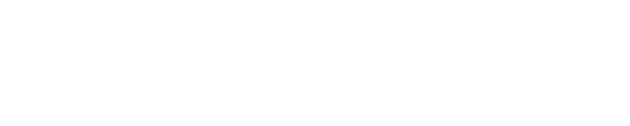

Jacqueline B Palmer'

Howard S Friedman ${ }^{2}$

Katherine Waltman

Johnson'

Prakash Navaratnam ${ }^{2}$

Stephen S Gottlieb ${ }^{3}$

'Novartis Pharmaceuticals Corporation, East Hanover, NJ, USA; ${ }^{2}$ DataMed Solutions, LLC, New York, NY, USA; ${ }^{3}$ Department of Medicine, University of Maryland School of Medicine, Baltimore, MD, USA
Correspondence: Stephen S Gottlieb Department of Medicine, University of Maryland School of Medicine, 110 South Paca Street, Baltimore, MD 21201 , USA

Tel + I 4103288788

Fax +14103281048

Email sgottlie@medicine.umaryland.edu
Background: Data comparing effects of transient worsening renal function (WRFt) and persistent WRF (WRFp) on outcomes in patients hospitalized with acute heart failure (AHF) are lacking. We determined the characteristics of hospitalized AHF patients who experienced no worsening renal function (non-WRF), WRFt, or WRFp, and the relationship between cohorts and AHF-related outcomes.

Methods and results: A patient's first AHF hospitalization (index) was identified in the Cerner Health Facts ${ }^{\circledR}$ database (January 2008-March 2011). Patients had WRF if serum creatinine (SCr) was $\geq 0.3 \mathrm{mg} / \mathrm{dL}$ and increased $\geq 25 \%$ from baseline, and they were designated as WRFp if present at discharge or WRFt if not present at discharge. A total of 55,436 patients were selected (non-WRF $=77 \%$, WRFp $=10 \%, \mathrm{WRFt}=13 \%$ ). WRFp had greater comorbidity burden than WRFt. At index hospitalization, WRFp patients had the highest mortality, whereas WRFt patients had the longest length of stay (LOS) and highest costs. These trends were observed at 30, 180, and 365 days postdischarge and confirmed by multivariable analyses. WRF patients had more AHF-related readmissions than non-WRF patients. In sensitivity analyses of the patient subset with live index hospitalization discharges, postdischarge LOS and costs were highest in WRFt patients, whereas mortality associated with a HF hospitalization was significantly higher for WRF patients vs non-WRF patients, with no difference between WRFp and WRFt.

Conclusion: In patients hospitalized for AHF, WRFp was associated with the highest mortality, whereas WRFt was associated with the highest LOS and costs. WRF patients had higher readmissions than non-WRF patients. Transient increases in SCr appear to be associated with detrimental outcomes, especially longer LOS and higher costs.

Keywords: renal function, acute heart failure, mortality rate, health outcomes, serum creatinine, cost

\section{Introduction}

The development of worsening renal function (WRF) occurs in approximately $25 \%$ of patients hospitalized for acute heart failure (AHF). ${ }^{1,2}$ Several studies have demonstrated that WRF in AHF patients is associated with increased mortality, length of stay (LOS), heart failure (HF)-associated hospital readmissions, and cost. ${ }^{2-7}$ A meta-analysis of 23 cohort, registry, and randomized controlled studies found that AHF patients with WRF had almost a 2-fold higher rate of all-cause mortality than those without WRF (unadjusted OR [odds ratio]: 1.75-95\% CI [confidence interval]: $1.47-2.08, P<0.001){ }^{1}$ 
Previous studies have used varying definitions of WRF, and populations have generally been limited to small groups of selected patients or have had limited access to complete data and follow-up. Furthermore, previous studies have rarely distinguished between transient WRF (WRFt) and persistent WRF (WRFp). The three studies that have directly compared outcomes associated with WRFt vs WRFp in patients hospitalized for AHF had somewhat contradictory results. ${ }^{7-9}$ One study found that 6-month postdischarge events (combined all-cause mortality or AHF-related readmission) were increased in patients with WRFt and WRFp. ${ }^{7}$ The second study found that only WRFp led to a significant increase in all-cause mortality at 6 months. ${ }^{8}$ In the third study, both WRFt and WRFp were associated with a higher risk of 90-day all-cause mortality, but the risk was significantly higher with WRFp vs WRFt. ${ }^{9}$ Similar to many other studies, these studies had different methods of defining WRF, and only one of the studies assessed LOS. None of the studies assessed cost outcomes based on WRF persistence.

Using contemporary data from a large multicenter database, the current analysis assessed short- and long-term AHF-related outcomes by WRF status using a rigorous clinically-based definition for WRF. The objective of this retrospective cohort analysis was to describe the characteristics of patients hospitalized for AHF who experienced no WRF (non-WRF), WRFt, or WRFp and to determine the association of these groups with in-hospital, 30-, 180-, and 365-day postdischarge mortality, risk of AHF readmission, LOS, and costs.

\section{Methods}

\section{Study design}

This was a retrospective longitudinal database analysis with a study period between January 2008 and March 2012. The index hospitalization was defined as the earliest valid inpatient hospital admission for AHF during the study period; index hospitalizations were determined up to March 30, 2011, and readmissions were tracked until March 30, 2012. AHF diagnosis was identified as hospitalization with a primary or secondary discharge International Classification of Diseases, 9th Edition (ICD-9) code for AHF (428.xx, 398.91, 402.x1, 404.x1, 404.x3, 415.0, 416.x, 417.x, 425.x, 429.0, 429.1 , or discharge diagnosis-related group of 127,291 , 292, and 293). These same diagnoses criteria were used for all assessed time points. The database used in the analysis was the Cerner Health Facts ${ }^{\circledR}$ (Kansas City, MO, USA) inpatient claims database, which contains deidentified patient data from Cerner and non-Cerner participating facilities.
Patient demographics, diagnoses, prescriptions, procedures, laboratory testing, hospital information, service location, and billing data from $>480$ contributing facilities throughout the United States were captured in the database (http:// www.bridgetodata.org/node/1789). The data set used in this analysis included patients with AHF-related admissions. All-cause outcomes were not explored in this study, since only AHF-related data were captured and tracked in the data set. At the index AHF-related hospitalization, mortality due to any cause was captured. Any mortality during a HF hospitalization was captured at the 30, 180, and 365-day post-discharge follow-ups.

\section{Patients}

To be included in the analysis, patients must have had $\geq 1$ record of an inpatient stay for AHF (primary or secondary diagnoses) between January 1, 2008, and March 30, 2011; had $\geq 3$ records of reported serum creatinine ( $\mathrm{SCr}$ ) values for the index hospitalization, including an admission (or baseline) SCr value within the same day or day after admission; and were $\geq 18$ years of age at the index hospitalization. Patients with any of the following were excluded from the analysis: record of elective or planned HF-related hospitalization, record of a heart transplantation procedure, diagnosis of chronic kidney disease stage 5 or end-stage renal disease requiring dialysis, diagnosis of cachexia or sarcopenia, record of death with LOS $\leq 1$ day at index hospitalization, missing cost or utilization data, unknown sex or age indicators, missing LOS data, or hospitalization for AHF within 1 year before the index hospitalization.

\section{Definition of WRF}

Eligible patients were divided into three renal function status cohorts based on SCr measurements during the index hospitalization. Currently, there is no expert consensus on how to define WRF. In the past, many studies have utilized a SCr cutoff relative to baseline..$^{2-5,10,11}$ However, there have been growing concerns that small changes in $\mathrm{SCr}$ could be associated with greater decreases in estimated glomerular filtration rate (eGFR). ${ }^{12}$ To adjust for this, some studies have explored using a combination of absolute $\mathrm{SCr}$ and $\mathrm{a} \geq 25 \%$ increase from baseline to define WRF. ${ }^{6,13}$ The current study will be the first to investigate outcomes such as LOS and costs using this more comprehensive definition of WRF. The last SCr measure before discharge was considered the discharge SCr. The WRFp cohort consisted of patients who had $\geq 0.3 \mathrm{mg} / \mathrm{dL}$ and $\mathrm{a} \geq 25 \%$ increase from baseline $\mathrm{SCr}$ that persisted at discharge of the index hospitalization. The 
WRFt cohort consisted of patients who had $\geq 0.3 \mathrm{mg} / \mathrm{dL}$ and $\mathrm{a} \geq 25 \%$ increase from baseline $\mathrm{SCr}$ that did not persist at discharge from the index hospitalization. The non-WRF cohort consisted of patients not identified as either WRFp or WRFt.

\section{Assessments}

Information at the index hospitalization was collected on patient demographics, hospital characteristics, comorbidities, and HF medication utilization. The primary analysis evaluated the relationship between WRF cohorts and mortality, LOS, and inpatient service costs for the index hospitalization and all subsequent AHF-related readmissions at cumulative postdischarge time points of 30,180 , and 365 days from the index hospitalization. In addition, the relationship between WRF cohorts and the number of AHF-related readmissions within 30,180 , and 365 days postdischarge from the index hospitalization was evaluated. Secondary analyses evaluated the relationship between AHF outcomes with index admission $\mathrm{SCr}$ and the index admission blood urea nitrogen (BUN)/ $\mathrm{SCr}$ ratio. Furthermore, the relationship between WRF status and eGFR threshold ( $<$ or $\geq 60 \mathrm{~mL} / \mathrm{min} / 1.73 \mathrm{~m}^{2}$ ) was determined.

\section{Statistical analyses}

Using the inclusion/exclusion criteria, a feasibility assessment determined that the sample size was large enough to ensure the study was well powered. Descriptive statistics of patient demographics, hospital characteristics, comorbidities, HF medication utilization, and secondary analyses were performed for comparison among WRF cohorts. Comparisons among all three cohorts (WRFt, WRFp, and non-WRF) and just two cohorts (WRFt and WRFp) were performed using Kruskal-Wallis or Wilcoxon Rank Sum for continuous variables and $\chi$-square test for categorical variables.

Multivariate modeling was conducted for the primary analysis. Covariates for all models were patient demographics, comorbidities, index admission $\mathrm{SCr}$, index admission $\mathrm{BUN} / \mathrm{SCr}$ ratio, hospital characteristics (including the number of beds, teaching status indicator, catheterization laboratory present indicator, and diagnostic catheterization laboratory indicator), and admission source and type. Logistic regression models were constructed to compare mortality among all three cohorts, or WRFt vs WRFp, at the index hospitalization, and mortality associated with all subsequent AHF-related readmissions within 30, 180, and 365 days of discharge. Negative binomial models were constructed to compare the number of AHF-related readmissions among all three cohorts, or WRFt vs WRFp, within 30, 180, and 365 days of discharge. Generalized linear models assuming gamma distribution were constructed to compare LOS and costs among all three cohorts, or WRFt vs WRFp, at the index hospitalization and all subsequent AHF-related readmissions within a cumulative (index + readmission) 30, 180, and 365 days of discharge. Costs were normalized to 2012 US dollars using the US Medical Care Services Consumer Price Index for All Urban Consumers.

\section{Sensitivity analyses and model diagnostics}

A sensitivity analysis was conducted to account for the potential impact of early mortality that could lead to biased estimates. This analysis used the same multivariate modeling described earlier for mortality, LOS, and costs, using only the subset of patients with a live discharge from the index hospitalization.

Regression diagnostics for the negative binomial and logistic regression models included an assessment of collinearity and influence diagnostics. Robustness analyses were conducted by constructing reduced models with significant variables only (retention fixed at $P<0.05$ ) and full models with outliers/heavy influence data points removed. The results of the robustness analyses were in agreement with those of the full analyses (data not shown). Analyses were conducted using the SAS software version 9.3 (SAS Institute Inc., Cary, NC, USA).

\section{Results \\ Patients}

The selection process identified 55,436 patients for inclusion in the analysis, with the most critical criterion being the requirement for $\geq 3$ records of $\mathrm{SCr}$ values (Figure 1). The majority of patients $(n=42,507,77 \%)$ were categorized as having non-WRF; $13 \%(n=7,366)$ and $10 \%(n=5,563)$ of patients were categorized as having WRFt and WRFp, respectively, with a total WRF prevalence of $23 \%$.

For the total population, the mean age was $72.4 \pm 14.3$ years, and $53 \%$ of patients were women. Patients with WRFp were older than patients with WRFt, but there was no difference in the sex distribution across cohorts (Table 1). Mean index admission SCr levels were similar among the cohorts, whereas the proportion of patients with eGFR of $<60 \mathrm{~mL} / \mathrm{min} / 1.73 \mathrm{~m}^{2}$ at admission was higher in the WRFp (53.9\%) and WRFt $(54.3 \%)$ patients compared with the non-WRF patients (51.4\%; Table 1). No meaningful conclusions could be made regarding differences among cohorts for vital signs, since these data were available only for a small percentage 


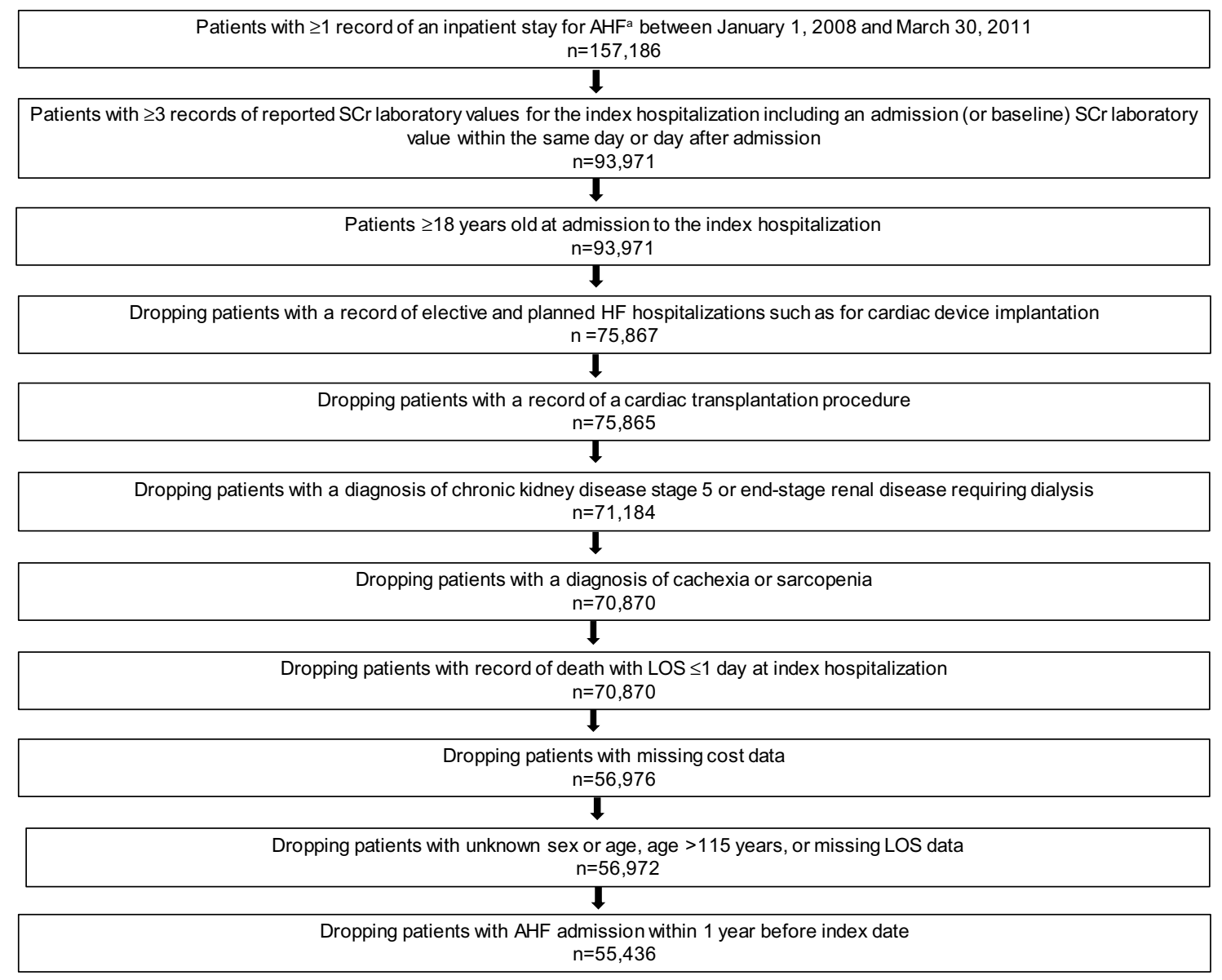

Figure I Patient selection.

Notes: ${ }^{2} \mathrm{An}$ AHF hospitalization is an admission having a primary or secondary diagnosis for any of the following ICD-9-CM heart failure codes: $398.9 \mathrm{I}, 402 . x \mathrm{I}, 404 . x \mathrm{I}, 404$. x3, 4I5.0, 4I6.x, 4I7.x, 425.x, 428.0, 428.I, 428.2x, 428.3x, 428.4x, 428.9, 429.0, 429.I.

Abbreviations: AHF, acute heart failure; HF, heart failure; LOS, length of stay; SCr, serum creatinine.

of patients. A higher overall comorbidity burden based on the Charlson Comorbidity Index was present in patients with WRFp compared to patients in the non-WRF and WRFt cohorts (Table 2). Patients with WRFp had significantly higher rates of cancer, sepsis, chronic liver disease, and impaired immune function, and lower rates of asthma and other respiratory conditions compared to patients with WRFt (Table 2). Non-WRF patients used fewer HF-related medications such as loop diuretics compared to patients with WRF. Patients with WRFp used less angiotensin-converting enzyme inhibitors, angiotensin receptor blockers, and $\beta$-blockers compared to patients with WRFt (Table 2).

\section{Mortality}

Patients with WRF had higher mortality rates than non-WRF at all time points (index, 30, 180, and 365 days postdischarge; Figure 2). The mean mortality rate at the index hospitalization was $23.6 \%, 5.7 \%$, and $3.9 \%$ for the WRFp, WRFt, and non-WRF cohorts, respectively (Figure 2). When compared with the non-WRF cohort in multivariate models, the OR for mortality during the index hospitalization was $7.76(95 \% \mathrm{CI}$ : $7.09-8.50 ; P<0.0001)$ in the WRFp cohort and 1.20 (95\% CI: 1.06-1.36; $P=0.0030$ ) in the WRFt cohort (Table 3). Patients in the WRFp cohort had greater than six times higher odds of mortality compared to those with WRFt (OR: 6.43; 95\% CI: 5.63-7.34) at the index hospitalization. The greater odds with WRFp vs WRFt persisted up to a year later when looking at death during a HF-associated hospitalization (Table 3). Other significant multivariable predictors for index hospitalization mortality and HF-associated hospitalization mortality included WRFp, sepsis, cardiogenic shock, pneumonia, index admission $\mathrm{SCr}$, and index admission BUN/SCr ratio. Most of the deaths (70\%) occurred during the index hospitalization, which drove the cumulative mortality results.

In the sensitivity analysis, in which only the subset of patients with live discharge from the index hospitalization were evaluated, patients in the WRFt cohort had significantly higher odds ratios for mortality associated with a HF 
Table I Patient and hospital characteristics by WRF cohort

\begin{tabular}{|c|c|c|c|c|c|c|}
\hline & $\begin{array}{l}\text { Total } \\
(n=55,436)\end{array}$ & $\begin{array}{l}\text { Non-WRF } \\
(n=42,507)\end{array}$ & $\begin{array}{l}\text { WRFp } \\
(n=5,563)\end{array}$ & $\begin{array}{l}\text { WRFt } \\
(n=7,366)\end{array}$ & 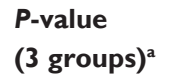 & $\begin{array}{l}\text { P-value } \\
\text { (WRFp vs WRFt) }\end{array}$ \\
\hline Age (year), mean (SD) & $72.4(14.3)$ & $72.3(14.4)$ & $73.5(13.5)$ & $71.9(13.9)$ & $<0.0001$ & $<0.0001$ \\
\hline Female (\%) & 53.1 & 53.2 & 52.4 & 53.4 & 0.4724 & 0.2411 \\
\hline Race (\%) & & & & & $<0.000$ I & $<0.0001$ \\
\hline White & 78.7 & 79.4 & 76.5 & 76.3 & & \\
\hline Black & 16.9 & 16.4 & 17.5 & 19.0 & & \\
\hline Hispanic & 1.2 & 1.2 & 1.3 & 1.4 & & \\
\hline Asian & 0.6 & 0.6 & 0.9 & 0.8 & & \\
\hline Unknown & 2.4 & 2.3 & 3.5 & 2.4 & & \\
\hline Index SCr (mg/dL), mean (SD) & I.4 (I.I) & $\mathrm{I} .5(\mathrm{I} .2)$ & I.4 (0.8) & I.4 (0.8) & 0.2306 & 0.1538 \\
\hline Index BUN/SCr ratio, mean (SD)c & $20.0(8.9)$ & $20.1(8.9)$ & $20.1(10.0)$ & $19.4(8.3)$ & $<0.0001$ & 0.0256 \\
\hline Index eGFR (\%) ${ }^{d}$ & & & & & 0.0009 & 0.733 \\
\hline$<60 \mathrm{~mL} / \mathrm{min} / \mathrm{l} .73 \mathrm{~m}^{2}$ & 52.0 & 51.4 & 53.9 & 54.3 & & \\
\hline$\geq 60 \mathrm{~mL} / \mathrm{min} / 1.73 \mathrm{~m}^{2}$ & 48.0 & 48.6 & 46.2 & 45.7 & & \\
\hline Number of hospital beds (\%) & & & & & $<0.0001$ & 0.0011 \\
\hline$<6$ & 0.03 & 0.02 & 0.02 & 0.1 & & \\
\hline $6-99$ & 8.1 & 8.5 & 7.5 & 6.4 & & \\
\hline $100-199$ & 15.7 & 16.1 & 14.4 & 13.9 & & \\
\hline $200-299$ & 18.8 & 19.0 & 19.1 & 17.9 & & \\
\hline $300-499$ & 26.2 & 26.6 & 24.9 & 24.4 & & \\
\hline $500+$ & 31.2 & 29.8 & 34.1 & 37.3 & & \\
\hline Hospital designation (\%) & & & & & 0.1066 & 0.7758 \\
\hline Rural & 0.2 & 0.2 & 0.1 & 0.1 & & \\
\hline Urban & 99.8 & 99.8 & 99.9 & 99.9 & & \\
\hline Hospital teaching status (\%) & & & & & $<0.0001$ & $<0.0001$ \\
\hline Non-teaching & 27.7 & 28.1 & 28.6 & 25.2 & & \\
\hline Teaching & 72.3 & 71.9 & 71.4 & 74.8 & & \\
\hline
\end{tabular}

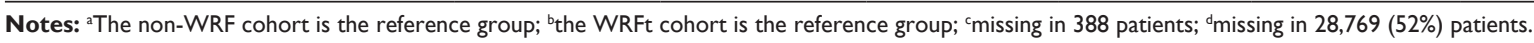

Abbreviations: BUN, blood urea nitrogen; eGFR, estimated glomerular filtration rate; SCr, serum creatinine; SD, standard deviation; WRF, worsening renal function; WRFp, persistent WRF; WRFt, transient WRF.

hospitalization at all time points than the reference non-WRF cohort ( $\mathrm{OR} \geq 1.24 ; P \leq 0.032$ ). There was a numeric trend toward higher odds ratios for mortality associated with a HF hospitalization for the WRFp cohort as well, but statistical significance was not always reached (OR $\leq 1.30 ; P \leq 0.146$ ). When patients with any WRF were combined together, they had significantly higher odds ratios for mortality associated with a HF hospitalization at 30,180 , and 365 days postdischarge compared to patients in the non-WRF cohort (OR $\geq 1.22 ; P<0.05)$. However, there were no significant differences in mortality associated with a HF hospitalization rates between the WRFp and WRFt cohorts at any time point in this mortality model, evaluating a subset of patients with live discharge from the index hospitalization.

\section{Length of stay}

Patients with WRFp had significantly shorter LOS at all time points compared with patients in the WRFt cohort, but had longer LOS compared with patients in the non-WRF cohort (Figure 3). The mean LOS at the index hospitalization was 10.2, 13.6, and 7.5 days for the WRFp, WRFt, and non-WRF cohorts, respectively (Figure 3). Compared with the non-WRF cohort in multivariate models, the index hospitalization LOS was significantly longer for the WRFp cohort $(P<0.0001)$ and for the WRFt cohort $(P<0.0001$; Table 3). Compared with the WRFp cohort, the index hospitalization LOS was significantly longer for the WRFt cohort $(P<0.0001$; Table 3$)$. This longer LOS for WRFt patients was observed at all time points (Table 3). Other significant multivariable predictors for LOS included WRFp, sepsis, pneumonia, acute cerebrovascular disease, WRFt, and cardiogenic shock. As with the mortality results, most of the inpatient stay (75\%) during the 365-day follow-up occurred during the index hospitalization, which drove the cumulative LOS results.

In the sensitivity analyses, the same LOS pattern (WRFt $>$ WRFp $>$ non-WRF) with respect to cohort was observed in patients with live index discharges and for the total population. Compared with the non-WRF cohort, the LOS was significantly longer for both WRFp and WRFt patients at all time points $(P<0.0001)$. Compared with the WRFp cohort, LOS was significantly longer for the WRFt cohort at all time points $(P<0.0001)$. 
Table 2 Medication utilization and comorbidities by WRF cohort

\begin{tabular}{|c|c|c|c|c|c|c|}
\hline & $\begin{array}{l}\text { Total } \\
(n=55,436)\end{array}$ & $\begin{array}{l}\text { Non-WRF } \\
(n=42,507)\end{array}$ & $\begin{array}{l}\text { WRFp } \\
(n=5,563)\end{array}$ & $\begin{array}{l}\text { WRFt } \\
(n=7,366)\end{array}$ & $\begin{array}{l}\text { P-value } \\
{\text { ( } 3 \text { groups })^{\mathrm{a}}}\end{array}$ & $\begin{array}{l}\text { P-value } \\
\text { (WRFp vs WRFt) }\end{array}$ \\
\hline \multicolumn{7}{|l|}{ HF-related medication use (\%) } \\
\hline ACE inhibitor & 42.7 & 41.3 & 44.3 & 49.8 & $<0.0001$ & $<0.000$ I \\
\hline ARB & 11.5 & 11.0 & 12.1 & 13.8 & $<0.0001$ & 0.0064 \\
\hline$\beta$-Blocker & 77.9 & 76.6 & 80.8 & 83.2 & $<0.0001$ & 0.0005 \\
\hline Loop diuretic & 71.1 & 67.7 & 82.5 & 82.2 & $<0.0001$ & 0.6309 \\
\hline Charlson comorbidity index score (SD) & $2.2(1.9)$ & $2.2(1.9)$ & $2.5(2.0)$ & $2.3(1.9)$ & $<0.0001$ & 0.0011 \\
\hline \multicolumn{7}{|l|}{ Comorbidities (\%) } \\
\hline Chronic liver disease and cirrhosis & 3.1 & 3.0 & 3.9 & 3.0 & 0.0015 & 0.0094 \\
\hline Valvular heart disease & 24.1 & 23.8 & 24.0 & 25.7 & 0.0024 & 0.0265 \\
\hline Other chronic respiratory conditions & 23.4 & 24.0 & 20.1 & 22.3 & $<0.0001$ & 0.0036 \\
\hline $\begin{array}{l}\text { Chronic respiratory failure, primary pulmonary } \\
\text { hypertension, and cardiopulmonary obesity }\end{array}$ & 21.6 & 22.1 & 18.6 & 20.6 & $<0.0001$ & 0.0043 \\
\hline Chronic impaired immune function & 14.0 & 13.7 & 16.0 & 13.9 & $<0.0001$ & 0.0010 \\
\hline Cancer & 10.0 & 10.0 & 11.0 & 8.9 & 0.0003 & $<0.0001$ \\
\hline Sepsis/septic shock & 8.0 & 6.7 & 13.3 & 11.5 & $<0.0001$ & 0.0021 \\
\hline Asthma & 8.0 & 8.0 & 7.0 & 8.3 & 0.0155 & 0.0063 \\
\hline
\end{tabular}

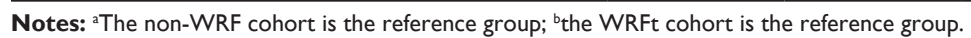

Abbreviations: ACE, angiotensin-converting enzyme; ARB, angiotensin receptor blockers; HF, heart failure; SD, standard deviation; WRF, worsening renal function; WRFp, persistent WRF; WRFt, transient WRF.

\section{Costs}

Patients with WRFp had significantly lower costs at all time points compared to patients with WRFt, but higher costs compared to patients in the non-WRF cohort (Figure 4). The mean index hospitalization costs were $\$ 29,252, \$ 38,412$, and $\$ 20,137$ for the WRFp, WRFt, and non-WRF cohorts, respectively (Figure 4). Compared with the non-WRF cohort in multivariate models, the index hospitalization costs were significantly higher for the WRFp cohort $(P<0.0001)$ and for the WRFt cohort $(P<0.0001$; Table 3). Compared with the WRFp cohort, the index hospitalization costs were significantly higher for patients in the WRFt cohort $(P<0.0001$; Table 3$)$. Similar results were seen for the other time points (Table 3 ). Other significant multivariable predictors for cost included WRFt, sepsis, cardiogenic shock, pneumonia, emergency admission, geographic region, and index year. As with mortality and LOS, most of the costs (78\%) occurred during the index hospitalization, which drove the cumulative cost results.

In the sensitivity analyses, the same cost pattern (WRFt $>$ WRFp $>$ non-WRF) with respect to cohort was observed in patients with live index discharges and for the total population. Compared with the non-WRF cohort, the

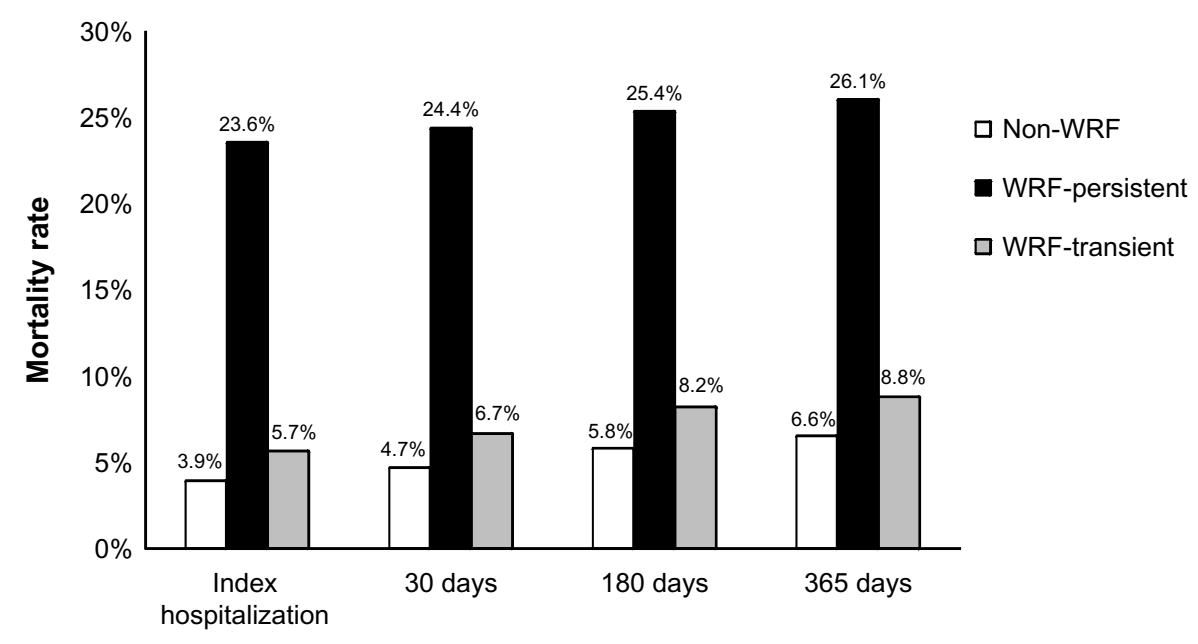

Figure 2 Mortality rate by WRF cohort.

Notes: Patients in the WRFp cohort had significantly $(P<0.000 \mathrm{I})$ higher rates of mortality at all time points than patients in the non-WRF and WRFt cohorts. Abbreviations: HF, heart failure; WRF, worsening renal function; WRFp, persistent WRF; WRFt, transient WRF. 
Table 3 Statistical modeling summary

\begin{tabular}{|c|c|c|c|c|c|c|c|c|c|}
\hline \multirow[t]{3}{*}{ Outcome } & \multirow[t]{3}{*}{ Comparison } & \multicolumn{8}{|c|}{ Coefficients/odds ratio } \\
\hline & & \multicolumn{2}{|c|}{$\begin{array}{l}\text { Index } \\
\text { hospitalization }\end{array}$} & \multicolumn{2}{|c|}{ 30-day model } & \multicolumn{2}{|c|}{ 180-day model } & \multicolumn{2}{|c|}{ 365-day model } \\
\hline & & WRFp & $\overline{\text { WRFt }}$ & WRFp & $\overline{\text { WRFt }}$ & WRFp & $\overline{\text { WRFt }}$ & WRFp & WRFt \\
\hline Readmission ${ }^{\mathrm{a}}$ (negative & WRFp vs WRFt vs non-WRF & & & $0.216^{\mathrm{e}}$ & $0.237^{\mathrm{e}}$ & $0.132^{\mathrm{e}}$ & $0.168^{\mathrm{e}}$ & $0.115^{\mathrm{e}}$ & $0.138^{\mathrm{e}}$ \\
\hline binomial model) & WRFp vs WRFt ${ }^{c}$ & & & -0.020 & & -0.036 & & -0.031 & \\
\hline Mortality ${ }^{d}$ (logistic & WRFp vs WRFt vs non-WRF & $7.763^{e}$ & $1.202^{\mathrm{e}}$ & $6.537^{\mathrm{e}}$ & $1.225^{\mathrm{e}}$ & $5.331^{e}$ & $1.269^{\mathrm{e}}$ & $4.830^{\mathrm{e}}$ & $1.218^{\mathrm{e}}$ \\
\hline regression model) & WRFp vs WRFt ${ }^{c}$ & $6.428^{e}$ & & $5.427^{e}$ & & $4.292^{\mathrm{e}}$ & & $\left.4.07\right|^{\mathrm{e}}$ & \\
\hline \multirow[t]{2}{*}{ 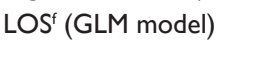 } & WRFp vs WRFt vs non-WRF & $0.211^{\mathrm{e}}$ & $0.489^{e}$ & $0.194^{\mathrm{e}}$ & $0.475^{e}$ & $0.163^{\mathrm{e}}$ & $0.447^{e}$ & $0.149^{e}$ & $0.429^{e}$ \\
\hline & WRFp vs WRFt ${ }^{c}$ & $-0.273^{\mathrm{e}}$ & & $-0.274^{e}$ & & $-0.278^{\mathrm{e}}$ & & $-0.273^{\mathrm{e}}$ & \\
\hline \multirow[t]{2}{*}{ Cost $^{g}$ (GLM model) } & WRFp vs WRFt vs non-WRF & $0.246^{\mathrm{e}}$ & $0.48 \mathrm{I}^{\mathrm{e}}$ & $0.227^{\mathrm{e}}$ & $0.464^{\mathrm{e}}$ & $0.195^{\mathrm{e}}$ & $0.443^{e}$ & $0.178^{e}$ & $0.425^{e}$ \\
\hline & WRFp vs WRFt ${ }^{c}$ & $-0.235^{\mathrm{e}}$ & & $-0.235^{\mathrm{e}}$ & & $-0.247^{\mathrm{e}}$ & & $-0.245^{\mathrm{e}}$ & \\
\hline
\end{tabular}

Notes: a Other significant multivariable predictors for readmission included WRFp, WRFt, atrial fibrillation, BUN/SCr ratio, index year, and admission source; bthe non-WRF cohort is the reference group; 'the WRFt cohort is the reference group; 'other significant multivariable predictors for mortality included WRFp, sepsis, cardiogenic shock, pneumonia, index admission $\mathrm{SCr}$, and index admission $\mathrm{BUN} / \mathrm{SCr}$ ratio; $\mathrm{P}<0.0$ I; fother significant multivariable predictors for LOS included WRFp, sepsis, pneumonia, acute cerebrovascular disease, WRFt, and cardiogenic shock; 'other significant multivariable predictors for cost included WRFt, sepsis, cardiogenic shock, pneumonia, emergency admission, geographic region, and index year.

Abbreviations: BUN, blood urea nitrogen; GLM, generalized linear model; LOS, length of stay; SCr, serum creatinine; WRF, worsening renal function; WRFp, persistent WRF; WRFt, transient WRF.

costs were significantly higher for both WRFp and WRFt patients at all time points $(P<0.0001)$. Compared with the WRFp cohort, costs were significantly higher for the WRFt cohort at all time points $(P<0.0001)$.

\section{Hospital readmissions}

Patients with WRFp and WRFt had a significantly higher number of AHF-related readmissions at all time points compared to patients in the non-WRF cohort; however, there was no difference between the WRFp and WRFt cohorts (Figure 5). The mean number of readmissions at 30 days postdischarge was $0.12,0.12$, and 0.09 for the WRFp, WRFt, and non-WRF cohorts, respectively (Figure 5). Compared with the non-WRF cohort in multivariate models, the readmission count at 30 days postdischarge was significantly higher for the WRFp cohort $(P<0.0001)$ and the WRFt cohort $(P<0.0001$; Table 3$)$. Other significant multivariable predictors for readmission included WRFp, WRFt, atrial fibrillation, BUN/SCr ratio, index year, and admission source. Nearly one-quarter (24\%) of the readmissions occurred during the first 30 days postdischarge.

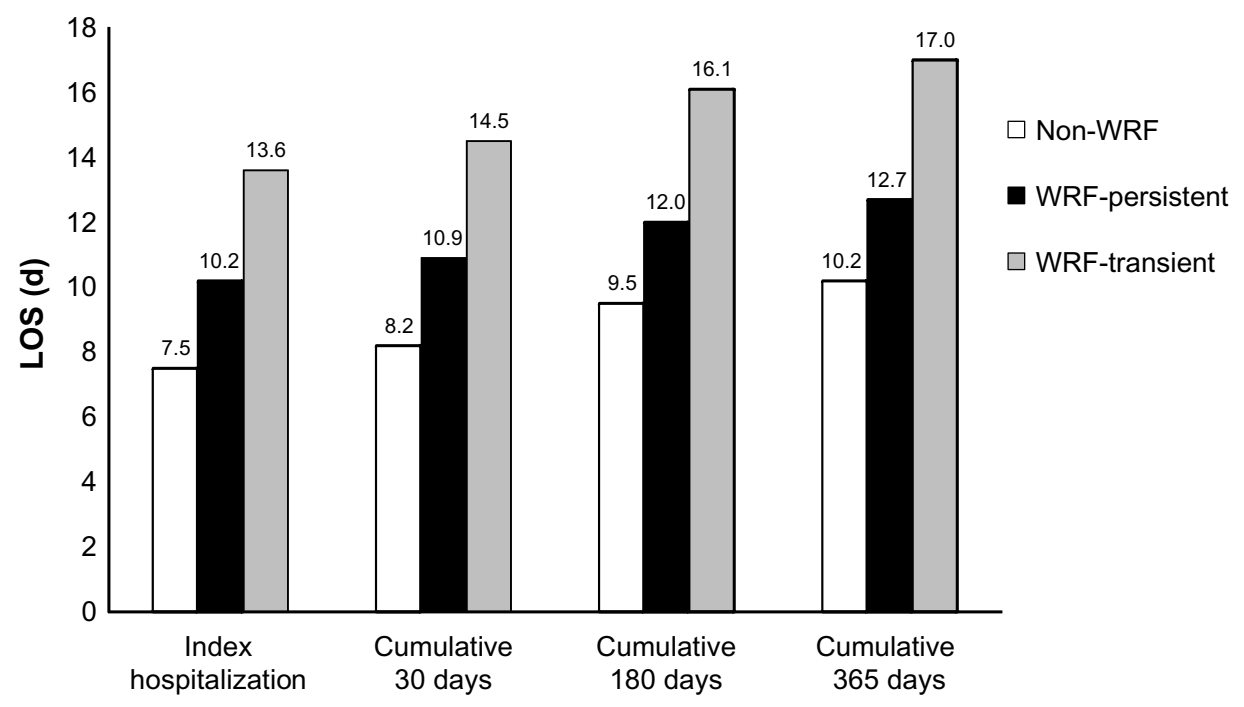

Figure 3 LOS by WRF cohort.

Notes: Patients in the WRFt cohort had significantly $(P<0.000 I)$ longer LOS at all time points than patients in the non-WRF and WRFp cohorts. The LOS was significantly longer in the WRFp cohort compared with the non-WRF cohort.

Abbreviations: LOS, length of stay; WRF, worsening renal function; WRFp, persistent WRF; WRFt, transient WRF. 


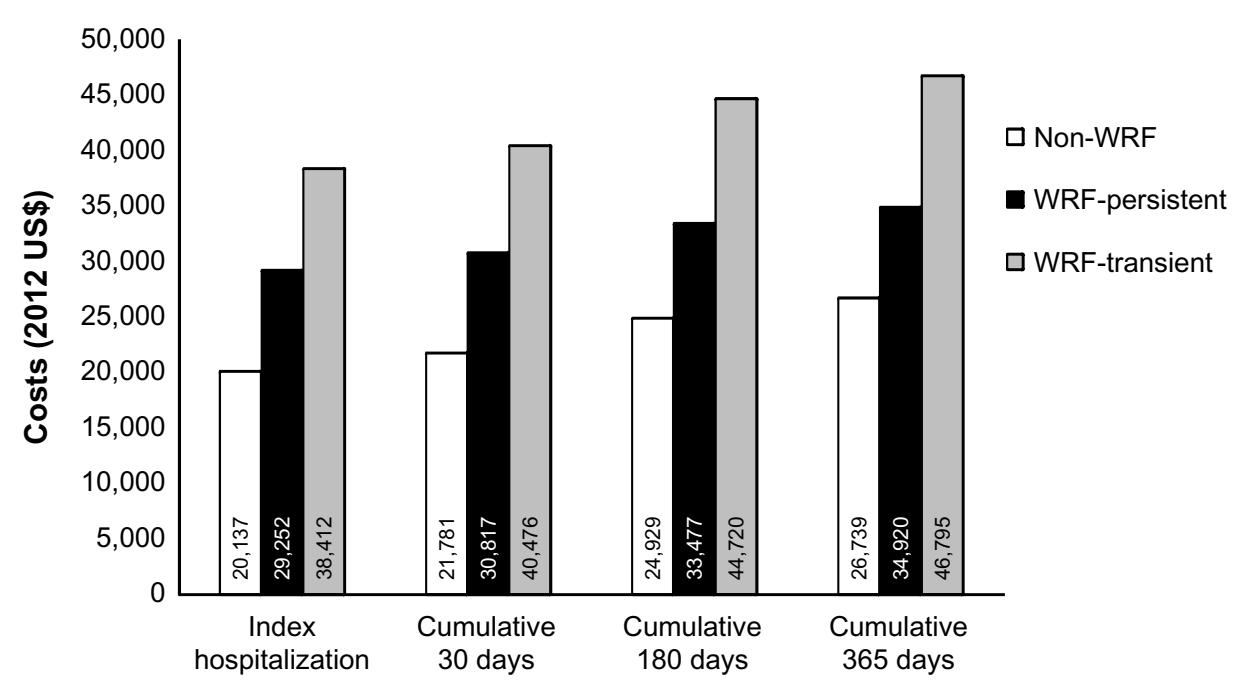

Figure 4 Cost by WRF cohort.

Notes: Patients in the WRFt cohort had significantly $(P<0.000 \mathrm{I})$ higher costs at all time points than patients in the non-WRF and WRFp cohorts. The costs were significantly higher in the WRFp cohort compared with the non-WRF cohort. Costs are in 2012 US dollars.

Abbreviations: WRF, worsening renal function; WRFp, persistent WRF; WRFt, transient WRF.

\section{Secondary analyses}

Results from the secondary analyses showed that there was no difference in the average index admission (within the same day or the day after admission) SCr among cohorts (Table 1), although the distribution was significantly different, with non-WRF having a higher percentage of patients at the extremes of higher and lower index admission levels. Index admission $\mathrm{SCr}$ was a significant predictor of mortality, LOS, and costs after controlling for other factors. The average index admission BUN/SCr ratio was significantly higher in the WRFp and non-WRF cohorts compared with the WRFt cohort (Table 1), and was a significant predictor for mortality, LOS, costs, and readmissions. The index admission eGRF was available only for $48 \%$ of patients. This parameter was significantly higher in the non-WRF cohort than in the WRFp and WRFt cohorts; however, there was no significant difference in the probability of belonging to the higher eGFR $\left(\geq 60 \mathrm{~mL} / \mathrm{min} / 1.73 \mathrm{~m}^{2}\right)$ group between the WRFp and WRFt cohorts (Table 1).

\section{Discussion}

In this retrospective analysis of patients hospitalized for AHF, patients in the WRFp cohort had significantly higher mortality rates compared with patients in the non-WRF and WRFt cohort, whereas patients in the WRFt cohort had significantly longer LOS and costs compared with patients in the non-WRF

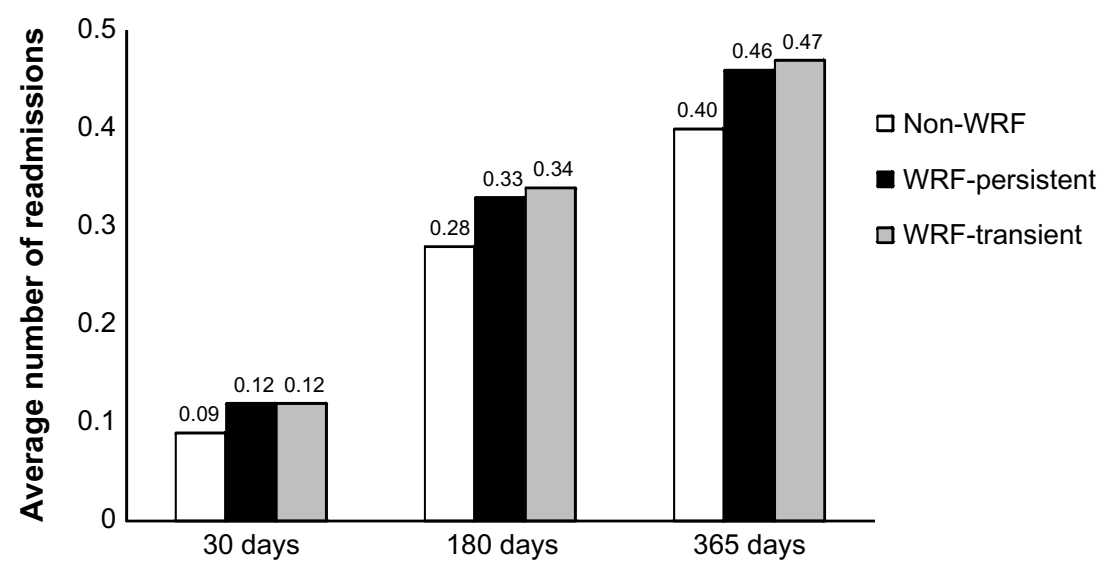

Figure 5 AHF-related hospital readmissions by WRF cohort.

Notes: Patients in the WRFt and WRFp cohorts had a significantly $(P<0.000 \mathrm{I})$ higher number of AHF-related readmissions at all time points than patients in the non-WRF. There was no significant difference in readmissions between the WRFt and WRFp cohorts.

Abbreviations: AHF, acute heart failure; WRF, worsening renal function; WRFp, persistent WRF; WRFt, transient WRF. 
and WRFp cohorts. Although any WRF was associated with a higher number of AHF-related readmissions, there was no difference between the WRFp and WRFt cohorts.

The mortality rate was strikingly higher in the WRFp cohort compared with the other cohorts. This finding may have been driven by the definitions used to characterize the WRF status cohorts. In 2002, Gottlieb et $\mathrm{al}^{11}$ demonstrated that a definition for WRF of change from baseline $\mathrm{SCr} \geq 0.3 \mathrm{mg} / \mathrm{dL}$ was both sensitive and specific for mortality and LOS. Since then, many studies have used this definition, but due to the exponential relationship between eGFR and $\mathrm{SCr}$, absolute changes in $\mathrm{SCr}$ are not equivalent in scale to changes in eGFR. ${ }^{12}$ To address this issue, we used an updated and more clinically rigorous definition for WRF that used both an absolute change in $\mathrm{SCr} \geq 0.3 \mathrm{mg} / \mathrm{dL}$ and a relative change in $\mathrm{SCr} \geq 25 \%$. $^{6}$ The use of this definition may have resulted in fewer patients being identified as having WRF. It is also possible that unmeasured factors that were not specifically addressed in this analysis (ie, concomitant chemotherapy, high- or low-protein diets, certain medications, etc) may have altered SCr levels, which would have affected WRF status.

Although the mortality rate was much higher in the WRFp cohort compared with the WRFt cohort, it is still notable that the mortality risk in the WRFt cohort was also significantly higher than the non-WRF cohort. This result is in agreement with that of a retrospective database study of Medicare patients reported by Krishnamoorthy et al, ${ }^{9}$ who found that both WRFt and WRFp (WRF defined as increase of SCr $\geq 0.3$ $\mathrm{mg} / \mathrm{dL}$ ) were associated with a significant risk of 90-day allcause mortality, although the risk with WRFp was $46 \%$ higher compared with WRFt. A study reported by Logeart et $\mathrm{al}^{7}$ also determined that both WRFt and WRFp (WRF defined as increase of $\mathrm{SCr} \geq 25 \mu \mathrm{mol} / \mathrm{L}$ [ $>0.28 \mathrm{mg} / \mathrm{dL}]$ ) in patients hospitalized for AHF were significantly predictive of worse outcomes (outcome of combined death and readmissions) 6 months postdischarge. Our findings on the increased risk of mortality in the WRFt cohort is in contrast to those of Aronson and Burger, ${ }^{8}$ who found that patients hospitalized for AHF with WRFt (WRF defined as increase of $\mathrm{SCr} \geq 0.5 \mathrm{mg}$ / dL) did not have significantly increased mortality 6 months postdischarge. In the current study, we also demonstrated that WRFt was associated with significantly higher LOS and costs compared with non-WRF and WRFp. These data indicate that negative outcomes are associated with WRFt and not just WRFp, and further investigation is needed for this population.

Patients in the WRFt and WRFp cohorts had higher rates of 30-day readmissions compared with patients in the
non-WRF cohort. In agreement with these results, in another retrospective claims database analysis of 20,063 patients, WRF was shown to increase the 30-day readmission risk in patients hospitalized for AHF. ${ }^{14}$ In contrast, a prospective study of 299 patients found no association between WRF and 30-day readmission in patients hospitalized for AHF. ${ }^{4}$ Per the Patient Protection and Affordable Care Act, Medicare reimbursement funds are decreased for hospitals with excessive 30-day readmission rates for AHF. The only other study that has investigated readmissions based on WRF persistence found no significant difference by adjusted analyses for either WRFt or WRFp in 90-day all-cause readmission or HF-related readmission. ${ }^{9}$ Although the published data seem contradictory, the discrepancies may be due to the slightly varying definitions used for WRF; the definition in the current study was more stringent than the other three studies that investigated readmissions, requiring both an absolute and percentage increase in SCr. The results of the current study add to previous studies by showing that WRF status may be a possible indicator of risk for 30-day readmission for AHF.

Most of the observed deaths, costs, and inpatient stay days occurred during the index hospitalization, which drove the cumulative results for the 30,180 , and 365 day time points. In sensitivity analyses that excluded patients who died during the index hospitalization, the presence of any WRF remained (postindex hospitalization) significantly associated with increased mortality associated with a HF hospitalization at all time points. However, although WRFp was highly associated with index hospitalization mortality, there was no longer a difference in postindex mortality associated with a HF hospitalization between the WRFp and WRFt cohort. The pattern of LOS and costs among cohorts in patients with live discharge remained the same as the total population (ie, significantly higher for patients in the WRFt cohort compared with the other cohorts), indicating that differences in mortality rates at the index hospitalization did not drive the LOS and cost results. The observation that cost and LOS were similar in the total population and patients with live discharge was not unexpected since 94\% of all patients had a live discharge. These data indicate that even transient increases in SCr appear to be associated with detrimental outcomes, such as longer LOS and higher costs when compared to patients with persistent increases. Post hoc analyses of a randomized, multicenter trial found that any change in renal function (either improved or worsened) with AHF was associated with longer LOS and increased mortality compared with stable renal function. ${ }^{15}$ Thus, any change in renal function, regardless of improved, worsened, 
transient, or persistent, appears to be associated with poor outcomes.

There are several strengths of this study including the use of data from a large multicenter database, the use of a stringent definition for WRF, and the assessment of both short- and long-term outcomes. Another notable strength of this study is the characterization of WRFt vs WRFp. The results of this study indicate that there is indeed a difference in outcomes based on persistence of WRF; however, both WRFp and WRFt were associated with negative outcomes.

In a meta-analysis of 16 studies, a significant linear relationship was found between SCr levels and mortality risk in patients with AHF. ${ }^{16}$ To determine if this relationship also applied to other biomarkers of renal function, we conducted secondary analyses of outcomes using index BUN/SCr ratio. After controlling for WRF status and other covariates, the index $\mathrm{BUN} / \mathrm{SCr}$ ratio appeared to be a strong predictor of all outcomes.

This study was limited by issues that apply to all retrospective database analyses, including the inability to determine and account for clinical factors, and the potential for miscoding of diagnoses or resource utilization procedures. The database is also limited, in that only data from patients that went to Cerner reporting hospitals were captured, and the majority of these reporting hospitals are teaching hospitals where the patient mix and practice patterns may be different than other hospitals. Furthermore, no data from hospitalizations outside the Cerner reporting hospitals were captured. An additional limitation is that only the inpatient experience of the patient was captured. Thus, if a patient died after discharge, additional data could not be captured and the patient was lost to follow-up. Furthermore, the data set used in this analysis was only for patients hospitalized for AHF; therefore, no data on all-cause outcomes were assessed, which may have shed some additional light on the impact on outcomes due to renal dysfunction. Finally, other AHF physiologic processes other than renal status that were not assessed in this study may be driving outcomes.

\section{Conclusion}

In patients hospitalized for AHF, WRFp was associated with the highest mortality rate, whereas WRFt was associated with the highest costs and LOS. WRF patients had higher readmission rates than non-WRF patients. Notably, transient increases in SCr appear to be associated with detrimental outcomes, especially longer LOS and higher costs. Further research is needed to understand the basis for these findings.

\section{Acknowledgments}

Medical writing and editorial assistance was provided by Erin P Scott, PhD, of Scott Medical Communications, LLC. This assistance and study were funded by Novartis Pharmaceuticals Corporation, East Hanover, NJ, USA. The study sponsor participated in the study design, interpretation of the data, and critical review of the manuscript. The final decision to submit the manuscript was made by the authors.

\section{Author contributions}

JBP, KWJ, and SSG contributed to the study design and interpretation of the data. HSF and PN contributed to data analysis and interpretation of the data. All authors participated in critically reviewing the manuscript for intellectual content, approved the final version for submission, and agreed to be accountable for all aspects of the work.

\section{Disclosure}

JBP and KWJ are employees of Novartis Pharmaceuticals Corporation, East Hanover, NJ, USA. HSF and PN are paid consultants for Novartis Pharmaceuticals Corporation. SSG received grant support from Novartis and has served as a consultant for Novartis, BMS, and Gambro. The authors report no other conflicts of interest in this work.

\section{References}

1. Damman K, Valente MA, Voors AA, et al. Renal impairment, worsening renal function, and outcome in patients with heart failure: an updated meta-analysis. Eur Heart J. 2014;35(7):455-469.

2. Forman DE, Butler J, Wang Y, et al. Incidence, predictors at admission, and impact of worsening renal function among patients hospitalized with heart failure. J Am Coll Cardiol. 2004;43(1):61-67.

3. Breidthardt T, Socrates T, Noveanu M, et al. Effect and clinical prediction of worsening renal function in acute decompensated heart failure. $\mathrm{Am} \mathrm{J}$ Cardiol. 2011;107(5):730-735.

4. Cowie MR, Komajda M, Murray-Thomas T, et al. Prevalence and impact of worsening renal function in patients hospitalized with decompensated heart failure: results of the prospective outcomes study in heart failure (POSH). Eur Heart J. 2006;27(10):1216-1222.

5. Herout PM, Harshaw Q, Phatak H, et al. Impact of worsening renal function during hospital admission on resource utilization in patients with heart failure. Am J Cardiol. 2010;106(8):1139-1145.

6. Metra M, Nodari S, Parrinello G, et al. Worsening renal function in patients hospitalised for acute heart failure: clinical implications and prognostic significance. Eur J Heart Fail. 2008;10(2):188-195.

7. Logeart D, Tabet JY, Hittinger L, et al. Transient worsening of renal function during hospitalization for acute heart failure alters outcome. Int J Cardiol. 2008;127(2):228-232.

8. Aronson D, Burger AJ. The relationship between transient and persistent worsening renal function and mortality in patients with acute decompensated heart failure. J Card Fail. 2010;16(7):541-547.

9. Krishnamoorthy A, Greiner MA, Sharma PP, et al. Transient and persistent worsening renal function during hospitalization for acute heart failure. Am Heart J. 2014;168(6):891-900. 
10. Amin AP, Spertus JA, Reid KJ, et al. The prognostic importance of worsening renal function during an acute myocardial infarction on long-term mortality. Am Heart J. 2010;160(6):1065-1071.

11. Gottlieb SS, Abraham W, Butler J, et al. The prognostic importance of different definitions of worsening renal function in congestive heart failure. J Card Fail. 2002;8(3):136-141.

12. Stevens LA, Coresh J, Greene T, Levey AS. Assessing kidney functionmeasured and estimated glomerular filtration rate. $N$ Engl J Med. 2006;354(23):2473-2483.

13. Damman K, Jaarsma T, Voors AA, et al. Both in- and out-hospital worsening of renal function predict outcome in patients with heart failure: results from the Coordinating Study Evaluating Outcome of Advising and Counseling in Heart Failure (COACH). Eur J Heart Fail. 2009;11(9):847-854.
14. Patel UD, Greiner MA, Fonarow GC, et al. Associations between worsening renal function and 30-day outcomes among Medicare beneficiaries hospitalized with heart failure. Am Heart J. 2010;160(1):132-138. e1.

15. Testani JM, McCauley BD, Kimmel SE, Shannon RP. Characteristics of patients with improvement or worsening in renal function during treatment of acute decompensated heart failure. Am J Cardiol. 2010;106(12):1763-1769.

16. Smith GL, Lichtman JH, Bracken MB, et al. Renal impairment and outcomes in heart failure: systematic review and meta-analysis. $J \mathrm{Am}$ Coll Cardiol. 2006;47(10):1987-1996.

\section{Publish your work in this journal}

ClinicoEconomics \& Outcomes Research is an international, peerreviewed open-access journal focusing on Health Technology Assessment, Pharmacoeconomics and Outcomes Research in the areas of diagnosis, medical devices, and clinical, surgical and pharmacological intervention. The economic impact of health policy and health systems organization also constitute important areas of coverage. The manuscript management system is completely online and includes a very quick and fair peer-review system, which is all easy to use. Visit http://www.dovepress.com/testimonials.php to read real quotes from published authors.

Submit your manuscript here: http://www.dovepress.com/clinicoeconomics-and-outcomes-research-journal 